\title{
UMA PROPOSTA DE ATIVIDADE PARA A APRENDIZAGEM SIGNIFICATIVA SOBRE AS FASES DA LUA
}

\author{
Bruno de Andrade Martins ${ }^{1}$ \\ Rodolfo Langhi ${ }^{2}$
}

\begin{abstract}
Resumo: Este texto foca um dos conteúdos de Astronomia e a consequente falha no ensino deste tema no ensino médio, apesar de os documentos oficiais apresentarem a necessidade de se trabalhar a Astronomia neste nível de ensino. Dentre as concepções alternativas em Astronomia que os alunos do ensino médio carregam consigo, mesmo após o término dos estudos, destacamos, nesta pesquisa, o fenômeno das fases da Lua. O desenvolvimento de estratégias diferenciadas em relação ao ensino tradicional, visando o processo de ensino-aprendizagem sobre este tema, foi contemplado neste trabalho como um dos resultados obtidos sob a luz dos referenciais da aprendizagem significativa, fundamentados em Ausubel. Segundo a proposta aqui apresentada, a participação ativa dos alunos na execução de uma atividade experimental e outras atividades didáticas, que visam sua contínua avaliação durante o processo, culmina com a elaboração de uma história em quadrinhos envolvendo as fases da Lua. Portanto, o objetivo deste trabalho é apresentar uma proposta de atividade didática diferenciada sustentada pelos princípios teóricos da aprendizagem significativa aplicada ao ensino das fases da Lua durante as aulas de Física no ensino médio.
\end{abstract}

Palavras-chave: Educação em Astronomia, Aprendizagem Significativa, Histórias em Quadrinhos, Atividades Experimentais.

\section{UNA ACTIVIDAD PROPUESTA PARA EL APRENDIZAJE SIGNIFICATIVO ACERCA DE LAS FASES DE LA LUNA}

Resumen: En este trabajo se presenta uno de los contenidos de la Astronomía y el evidente fracaso en la enseñanza del tema en la escuela secundaria, a pesar del hecho que los documentos oficiales apuntan para la necesidad de trabajar contenidos de Astronomía en este nivel. Entre los conceptos alternativos en Astronomía que los alumnos secundarios llevan consigo, aún después de terminados los estudios, destacamos aquí el fenómeno de las fases de la Luna. El desarrollo de diferentes estrategias en relación con los métodos tradicionales, dirigidas al proceso de enseñanza-aprendizaje en este tema fue considerado en este trabajo como uno de los resultados obtenidos a la luz de los referenciales del aprendizaje significativo, tal como fueron fundamentados por Ausubel. Según la propuesta que aquí se presenta, la participación activa de los estudiantes en la ejecución de una actividad experimental y otras actividades educativas destinadas a la evaluación continua durante el proceso culminó en la elaboración de una historieta respecto de las fases de la Luna. Por lo tanto, el objetivo de este trabajo es presentar una propuesta de actividad de enseñanza diferenciada con el apoyo de los principios teóricos del aprendizaje significativo aplicado a la enseñanza de las fases de la luna durante las clases de física del ciclo secundario.

Palabras clave: Enseñanza de la Astronomía, Aprendizaje Significativo, Historietas, Actividades experimentales.

\footnotetext{
${ }^{1}$ Mestrando da Universidade Federal de Mato Grosso do Sul (UFMS). Programa de Pós-Graduação em Ensino de Ciências. Grupo de Pesquisa "Ciências: Ensino e Popularização". Apoio: CAPES- REUNI. <martinsfisica@gmail.com>.

${ }^{2}$ Professor Assistente Doutor da UNESP/Bauru. Programa de Pós-Graduação em Ensino de Ciências. Grupo de Pesquisa em Ensino de Ciências. Observatório Didático de Astronomia UNESP. Apoio: Programa de fomento do PTI C\&T/FPTI-BR. <rlanghi@fc.unesp.br>.
} 


\title{
A PROPOSED ACTIVITY FOR A MEANINGFUL LEARNING ABOUT THE MOON PHASES
}

\begin{abstract}
This paper presents one of the concepts of Astronomy and its consequent failure in teaching this topic in high school, even when the official documents point out the necessity of Astronomy teaching at this school level. Among the spontaneous conceptions in Astronomy that high school students carry with them, even after the end of the school, we emphasized in this research the Moon phases. The development of different strategies in relation to traditional methods, aimed to teaching-learning process on this topic was considered in this study. These strategies were devised based on the reference frame of the Meaningful Learning, as elaborated by Ausubel. The proposals presented here include the active participation of students in experimental activities and other didactic activities, for their continuous evaluation during the process. These activities finished with a Comics elaboration about the Moon phases. Therefore, the objective of this paper is to present a proposal for differentiated teaching activity about Moon phases supported by the theoretical principles of Meaningful Learning at Physics classes.
\end{abstract}

Keywords: Astronomy Education, Meaningful Learning, Comics, Experimental Activity.

\section{Introdução}

A Astronomia, enquanto ciência que estuda os corpos celestes, é considerada uma das primeiras ciências estudadas pelo homem. Apesar de ser uma ciência antiga e fundamental para o entendimento de vários fenômenos observáveis e com ligações diretas com nosso cotidiano, observamos que atualmente, mesmo tendo seus conteúdos indicados nos Parâmetros Curriculares Nacionais (BRASIL, 1999, 2002), os alunos terminam o ensino médio sem o conhecimento de muitas questões na referida área, contribuindo fortemente para o despreparo do aluno frente ao estudo das ciências (LANGHI, 2009). Com esse despreparo, a Astronomia é pouco compreendida pelo público em geral, desde crianças a professores de todos os níveis de ensino, além do fato de não estar ocorrendo a inclusão dos conceitos de Astronomia na maioria dos currículos escolares (ALBRECHT e VOELZKE, 2009).

Segundo Kantor (2001), a Astronomia pode ser um tema com grande potencial para desenvolver a capacidade de observação, análise e interpretação de fenômenos naturais, uma vez que alguns acontecimentos astronômicos são de livre acesso à observação. O desenvolvimento destas capacidades potencializa-se com a construção de atividades práticas, as quais não necessariamente precisam ser aplicadas à noite, como demonstram alguns trabalhos da área (JACKSON, 2009; DARROZ, HEINECK e PÉREZ, 2011; ROS, 2009; MARRANGHELLO e PAVANI, 2011; LEÃO, 2011; LONGHINI, SILVESTRE e VIEIRA, 2010).

Assim, o estudo dos astros pode ser realizado sem instrumentos sofisticados, pois podemos observar seus fenômenos a olho nu, mesmo estes se encontrando a grandes distâncias, tornando-se o céu um "laboratório" astronômico (LANGHI, 2011). A Astronomia também tem uma influência direta em nossas vidas, como por exemplo: estações do ano, fases da Lua, dia e noite, contagem do tempo, construção de calendários, influências nas marés, orientações para navegações, satélites, GPS. Estes exemplos tornariam os conteúdos de Astronomia significativos para o aprendizado, sob à luz da fundamentação teórica utilizada neste trabalho (MOREIRA e MASINI, 1982).

O estudo da Astronomia, de caráter interdisciplinar, é apoiado por um dos documentos oficiais para a educação nacional, os PCN, os quais reconhecem que os assuntos a ela relacionados podem ser tratados em outras disciplinas (BRASIL, 1999 e 2002). Além disso, há a necessidade de reverter um quadro no qual se constata 
empiricamente uma grande difusão de concepções de senso comum referentes aos fenômenos astronômicos, além de a Astronomia promover um papel motivador, pois:

\begin{abstract}
Ao tocar neste assunto, a maioria dos jovens costuma desencadear uma enxurrada de perguntas sobre buracos negros, origem do universo, vida extraterrestre, tecnologia aeroespacial, etc. Este entusiasmo abre a oportunidade para o professor trabalhar, de modo interdisciplinar, as demais matérias escolares. Além do aspecto motivacional, a astronomia assume um papel diferenciador, que a pode distinguir das outras ciências, conferindo-lhe um certo grau "popularizável", favorecendo a cultura científica, uma vez que o seu laboratório é natural e gratuito, estando o céu à disposição de todos, facilitando a execução de atividades ao ar livre e que não exigem materiais custosos (LANGHI, 2009, p. 10).
\end{abstract}

Dentre os diversos conteúdos e temas que podem ser trabalhados em Astronomia, levamos em conta o levantamento efetuado por Langhi e Nardi (2010), que apresentam os sete conteúdos mais recorrentes na pesquisa nacional e nos documentos oficiais da educação (chamados de "astronomia essencial"): forma da Terra, fases da Lua, estações do ano, campo gravitacional, dia e noite, órbita terrestre e astronomia observacional. Este levantamento da produção nacional concorda com levantamentos internacionais, onde apontam também estes temas principais (PRICE, 2009; BAILEY e SLATER, 2004). No presente trabalho, optamos pela escolha de um destes, a saber, as fases da Lua. Outros autores já trabalharam este tema em suas investigações, constatando as concepções existentes em alunos e professores, algumas das quais sendo: a sombra da Terra provoca as fases da Lua, as nuvens são as responsáveis pelas fases da Lua, outros planetas provocam as fases da Lua, a Lua descreve sua órbita ao redor do Sol (IACHEL, LANGHI e SCALVI, 2007; ANDRADE et al., 2009; LANGHI e NARDI, 2010). Demais artigos podem auxiliar o professor no entendimento deste fenômeno lunar e o trabalho em sala de aula (DARROZ et al, 2012; QUEIROZ, SOUZA e MACHADO, 2009; SILVEIRA, 2001 e 2003; SARAIVA et al, 2007).

Diante da problemática acima apresentada, reconhecemos a necessidade de superação destas concepções por meio da proposta de uma atividade didática que aborde esta questão sob a visão da aprendizagem significativa. Assim, a questão central é: como trabalhar este tema no ensino médio, de modo que os alunos sintam-se motivados para uma aprendizagem significativa?

\title{
2. Referencial teórico
}

A Aprendizagem Significativa de David Ausubel leva em conta o processo através do qual uma nova informação interage com as informações já existentes na estrutura de conhecimento do aluno, ou seja, com a sua estrutura cognitiva específica e individual, previamente adquirida, conhecida como "subsunçores", facilitando a aprendizagem subsequente (MOREIRA e MASINI, 1982).

A cognição, segundo Moreira e Masini (1982), é o processo pelo qual o indivíduo estabelece relações, atribuindo significados à realidade que o cerca. Partindo destes significados, constroi-se a estrutura cognitiva, na qual se formam os primeiros conceitos, denominados pontos de ancoragem, nos quais novos significados são alicerçados e desenvolvidos. Esses pontos de ancoragem são chamados de subsunçores os quais são, segundo Ausubel, os conceitos preexistentes na estrutura cognitiva do indivíduo. 
O levantamento das concepções iniciais dos alunos sobre o tema a ser abordado é essencial para a aplicação de uma estratégia de ensino ou para a elaboração de um material didático, a fim de que o professor tente superar as concepções alternativas que os alunos porventura tenham. Por fim, deve-se efetuar a verificação posterior desta superação a fim de se constatar se as concepções iniciais dos alunos foram superadas devido à estratégia abordada, ou seja, verificar se a estratégia aplicada atuou como uma "ponte" para a sua estrutura cognitiva. Deste modo, os resultados podem fornecer indícios de uma aprendizagem significativa nos alunos. No presente estudo, estamos especialmente interessados em uma aprendizagem significativa sobre as fases da Lua.

\begin{abstract}
Os conceitos pré-existentes são muito importantes, pois podem servir de alicerce e facilitadores na aprendizagem de temas e conceitos correlacionados. Porém, o ensino de Física na maior parte dos casos, acontece apenas como uma reprodução de fórmulas sem considerar os conhecimentos prévios que estão ligados na realidade do educando (ALBRECHT e VOELZKE, 2009).
\end{abstract}

A teoria de Ausubel ainda destaca dois interessantes processos que ocorrem na Aprendizagem Significativa: a diferenciação progressiva e a reconciliação integrativa. A diferenciação progressiva está relacionada com a aprendizagem subordinada e ocorre quando um conceito subsunçor, através de sucessivos processos de ancoragem, sofre modificações de significado, diferenciando-se progressivamente, adquirindo deste modo maior estabilidade e clareza (MOREIRA e MASINI, 1982). A reconciliação integrativa ocorre quando, na aprendizagem superordenada ou combinatória, ideias presentes na estrutura cognitiva são reconhecidas como relacionadas, a partir de um processo de interação entre elas, podendo reorganizar-se esta estrutura e adquirir novos significados (ROSA, 2008).

Para que ocorra a aprendizagem significativa são necessárias duas condições, segundo Pelizzari et al. (2002): a) o aluno precisa estar disposto a aprender: se ele simplesmente quiser memorizar o conteúdo de maneira literal e arbitrária, a aprendizagem será mecânica; b) o conteúdo deve ser lógico e psicologicamente significativo: o significado lógico depende somente da natureza do conteúdo, e o significado psicológico é uma experiência que cada indivíduo tem. Cada aprendiz faz uma filtragem dos conteúdos que têm significado ou não para si próprio.

Partindo destes princípios teóricos fundamentadores, uma atividade experimental atuaria como esta "ponte" para a estrutura cognitiva do aluno, desde que adequadamente trabalhados seus subsunçores sobre o tema a ser desenvolvido em sala de aula.

Pelo fato de a literatura da área indicar as potencialidades motivacionais da inserção da Astronomia no ensino, optamos por um dos seus inúmeros conteúdos, a saber, as fases da Lua, uma vez que tal fenômeno é parte do cotidiano dos alunos e isto representaria, a princípio, uma pré-disposição a aprender sobre este fenômeno, segundo a fundamentação sobre a qual nos apoiamos. Além disso, a fim de diferenciar a aplicação desta proposta, sob um viés contrário ao da memorização e do ensino meramente conteudista, optamos pela construção e utilização de um material didático que reproduz um modelo das fases da Lua. De fato, de acordo com Camino (2004), estes tipos de "modelos concretos" funcionam como ferramentas para a aprendizagem de Astronomia desde que seguidos alguns critérios ao elaborá-los. Além disso, propomos a produção textual pelos próprios alunos, os quais exerceriam uma função de 
autoria de Histórias em Quadrinhos, cuja estratégia funcionaria também como um método de avaliação do processo (TESTONI e ABIB, 2005). A produção das Histórias em Quadrinhos tem por objetivo promover a reconciliação integrativa, segundo a teoria de Ausubel, fazendo com que o indivíduo reorganize seus conceitos estabelecendo vínculos e ligações entre conceitos menos inclusivos e conceitos mais inclusivos (MARTINS, 2010).

Portanto, sob o referencial adotado, este trabalho visa apresentar uma proposta de aplicação de um material didático experimental no ensino de Astronomia para a Aprendizagem Significativa sobre as fases da Lua. Como objetivos específicos desta proposta, temos: investigar as concepções dos alunos acerca do tema fases da Lua; demonstrar aos alunos como ocorrem as fases da Lua por meio do protótipo (material didático); investigar como os alunos do terceiro ano do ensino médio se apropriam dos conceitos estudados durante a sequência didática (em aulas de Física); investigar indícios de uma aprendizagem significativa dos alunos em suas escritas, conceitos, desenhos etc. que estarão presentes em suas Histórias em Quadrinhos.

Acreditamos que o tema abordado sobre as fases da Lua, o material didático construído e as Histórias em Quadrinhos sejam métodos que satisfazem as condições para uma aprendizagem significativa, segundo Ausubel (MOREIRA e MASINI, 1982), conforme já apontam alguns trabalhos neste sentido (MARTINS, 2010; ALBRECHT e VOELZKE, 2009; TESTONI E ABIB, 2005; WERLANG; SCHNEIDER e SILVEIRA, 2008; MARTINS e LANGHI, 2011).

\section{A proposta}

A metodologia empregada neste trabalho está focada na abordagem qualitativa, visando a produção de um material didático de apoio ao trabalho em sala sobre o tema, fundamentada nos referenciais da aprendizagem significativa, conforme apresentados acima. A proposta é que o próprio professor seja capacitado a construir um aparato que sirva de modelo para simular o sistema Terra-Lua (CAMINO, 2004). O objetivo do uso desse material didático é proporcionar aos alunos uma aula diferenciada em relação às aulas tradicionais e meramente conteudistas, em que os professores não utilizam nenhum outro tipo de recurso diferente do quadro e giz. Segundo Albrecht e Voelzke (2009), as aulas de Física são geralmente ministradas de maneira excessivamente expositiva em sala de aula, ou seja, predomina-se o ensino tradicional, enfatizando a matemática e o uso de equações, o que ocasiona um rápido desinteresse pelo aprendizado da Física. Segundo Werlang, Schneider e Silveira (2008), um material didático utilizando modelagens (modelos didáticos) pode facilitar o processo de ensinoaprendizagem, desde que seja desenvolvido de modo contextualizado com a realidade dos aprendizes, o que se leva em conta nesta proposta.

A produção das Histórias em Quadrinhos, ao longo do uso deste aparato experimental que modela as fases da Lua, proporciona um meio de avaliação contínua de todo o processo e dos participantes. Optamos em trabalhar com esse recurso, pois, além de seu caráter popular, as Histórias em Quadrinhos apresentam, do ponto de vista pedagógico, uma série de fatores coerentes com seu uso didático: a ludicidade, os fatores psicolinguísticos e seu aspecto cognitivo (TESTONI e ABIB, 2005). 
As Histórias em Quadrinhos veem, há mais de um século, influenciando várias gerações, e seu formato popular e de fácil leitura transforma esta forma de arte em um objeto de aprendizagem e lazer. O fato de estarmos tratando de um instrumento de caráter cotidiano, de cunho popular e fácil interpretação, aliado com as demais características relacionadas aos aspectos lúdicos, linguísticos e psicológicos dos desenhos e da narrativa, leva-nos a considerar os Quadrinhos como uma estratégia potencial para o ensino de Ciências, como já atestado por pesquisas anteriores (TESTONI, 2004; GONÇALVES e MACHADO, 2005; KAMEL, 2006; VÍLCHEZGONZÁLEZ e PERALES-PALACIOS, 2006; WORNER e ROMERO, 1998).

Os alunos podem produzir as Histórias em grupos, interagindo com o trabalho do material didático que modela as fases da Lua. A utilização de Histórias em Quadrinhos elaboradas pelos alunos pode ser também uma estratégia para se trabalhar suas concepções em sua estrutura cognitiva, potencializando-os a buscarem mais ferramentas e conhecimentos com apoio nos conceitos da Física e consequentemente construir suas Histórias usando conceitos cientificamente aceitos (TESTONI, 2004; MARTINS, 2010). De fato, a reconciliação progressiva, segundo Ausubel (MOREIRA e MASINI, 1982) e a organização de novos conhecimentos podem ser potencializadas com a autoração de Histórias em Quadrinhos, pois os alunos desenvolvem habilidades e competências para exemplificar e enunciar de modo satisfatório os conceitos envolvidos no ensino e trabalhados em sala, podendo relacioná-los com seu cotidiano ao elaborar um enredo. Isto poderia indicar uma assimilação de conceitos em sua estrutura cognitiva, modificando os conceitos prévios que os mesmos possuíam antes da produção das Histórias, conforme já constatado por outros trabalhos, como em Testoni e Abib (2005).

Assim, como um dos resultados preliminares deste estudo, apresentamos a proposta de construção do modelo que simula as fases da Lua, fundamentada nos referenciais teóricos acima apresentados. Os materiais necessários para sua montagem são: uma bola de isopor de $10 \mathrm{~cm}$ de diâmetro; uma bola de isopor de $2,5 \mathrm{~cm}$ de diâmetro; aro de bordar; um suporte circular de madeira; cola; um pequeno suporte de ferro; arame grosso; uma lanterna; um pequeno suporte de madeira circular de diâmetro de $18 \mathrm{~cm}$. Os tamanhos da Lua e da Terra, representados pelas esferas de isopor, estão em escala, embora a distância entre estes astros não estejam, pois a construção do modelo se tornaria inviável para a proposta aqui apresentada e fugiria dos objetivos de simular as fases da Lua, uma vez que as esferas estariam distanciadas entre si de quase três metros.

$\mathrm{O}$ aro de bordar representa a órbita que a Lua realiza em torno da Terra e por onde a bola de isopor vazada (Lua) irá orbitar, mostrando assim as fases da Lua. O suporte de ferro representa o eixo de rotação da Terra, os dois suportes de madeira irão sustentar o protótipo e a lanterna fará o papel do Sol. Este modelo, mostrado na Figura 1, dá suporte para o professor explicar os motivos pelos quais os eclipses não ocorrem em toda a Lua Cheia e Luz Nova, ao usar o aro inclinado, simulando a órbita lunar e a linha dos nodos. A presente discussão, porém, foca especificamente as fases da Lua, de modo que os eclipses seriam um tema para aulas posteriores. 


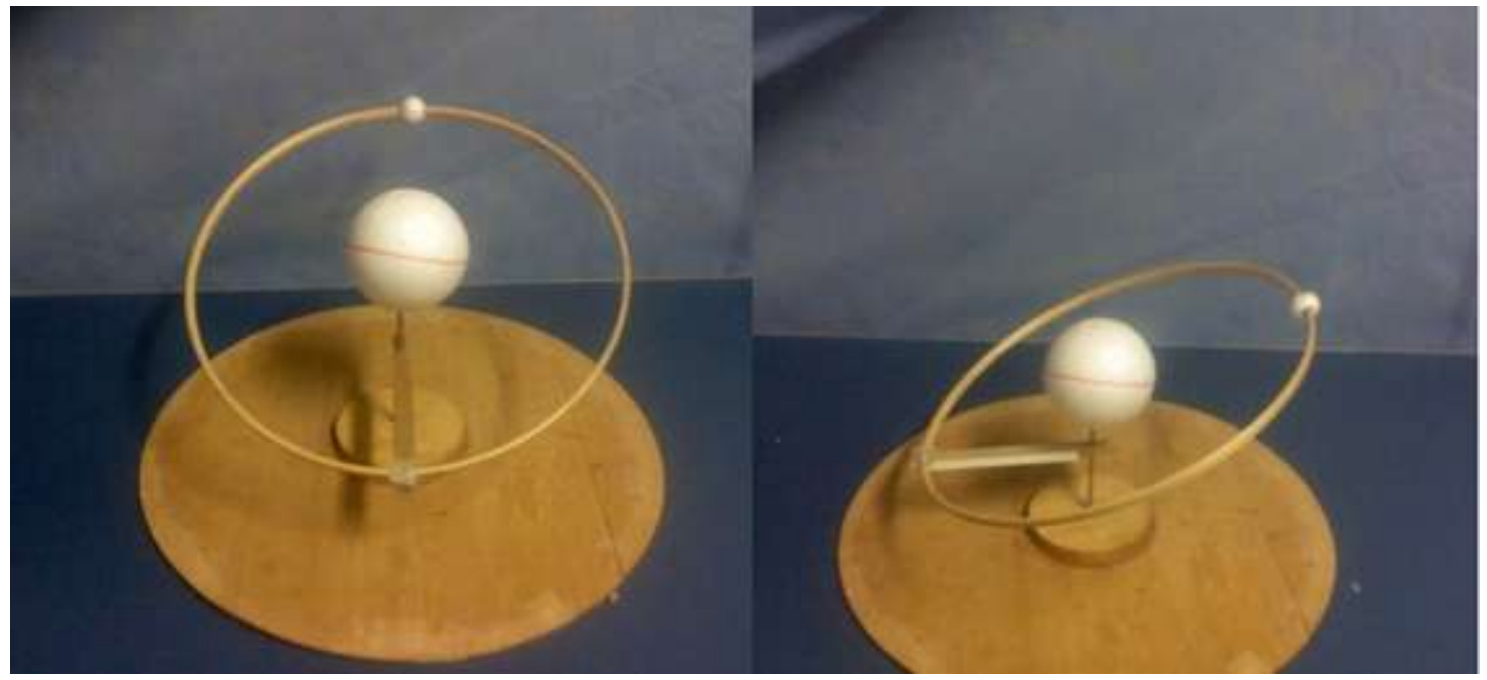

Figura 1: Simulador do sistema Terra/Lua

A proposta, ainda não aplicada, adota uma sequência didática seguindo os padrões de Ausubel com foco no protótipo (material didático). Nesta teoria, os conhecimentos prévios dos estudantes deixam de ser um problema e passam a ser ponto de partida para a construção dos novos conceitos (MOREIRA e MASINI, 1982).

Primeiramente, levantam-se as concepções iniciais dos alunos sobre as fases da Lua. Tal ação pode ser realizada por meio do uso de questionários apropriados ou com dinâmicas de grupo, conforme exemplos de instrumentos encontrados e já aplicados em pesquisas anteriores (LONGHINI, 2010). Daí, pelo uso do próprio material didático construído pelo professor, solicita-se aos mesmos que organizem o sistema Terra-Lua de modo que eles consigam demonstrar como ocorrem as fases da Lua, sendo questionados sobre tal pensamento. Parte-se destas concepções levantadas a fim de progressivamente se construir o conhecimento acerca deste tema. Posiciona-se o sistema Terra-Lua de acordo com as respostas dos alunos, e em seguida os próprios constatam se seus argumentos validam-se mediante a simulação. Durante todo o processo nas aulas, estas discussões devem ser representadas por eles em Histórias em Quadrinhos elaboradas por grupos de três alunos, pois o trabalho em grupo permite:

\begin{abstract}
Um processo fundamentalmente de comunicação, os alunos interagem entre eles e com o professor, de forma ativa, portanto, diferenciada de quando somente o professor tem a palavra, durante as aulas. Com isso queremos dizer que nos grupos de ensino-aprendiz agem, criados durante as aulas de Física podem contribuir "permitindo ao aluno se comunicar, debater sua compreensão, aprender a respeitar e a fazer-se respeitar; dando ao aluno oportunidade de construir modelos explicativos, linhas de argumentação e instrumentos de verificação de contradições (SILVA e VILLANI, 2008).
\end{abstract}

Tais produções servem de base para a análise de seus discursos, conceitos e representações usadas para explicar as fases da Lua, o que permite a verificação de indícios de uma aprendizagem significativa pelos alunos. Neste sentido, é importante os alunos organizarem seus pensamentos, pois:

Ao construir o próprio conhecimento é possível que o aluno consiga fazer modificações em seu comportamento. Isso remete à aprendizagem e está ligado também à mudança de paradigma, de construção de uma nova 
verdade. Uma verdade aceita e construída de maneira que o educando possa entender que os conceitos científicos são construídos pelos seres humanos, ou seja, perceber que a Ciência é uma construção humana. (MARTINS, 2010).

As Histórias em Quadrinhos produzidas em sala podem livremente abordar qualquer tipo de personagem ou sequência desde que envolvam as fases da Lua. $\mathrm{O}$ tempo de execução previsto para esta sequência didática é de quatro horas-aula. Um dos objetivos dessa estratégia é fazer com que o professor sirva de mediador para os grupos apreenderem os conceitos cientificamente aceitos sobre as fases da Lua, numa tentativa de tornar a Física mais "atrativa" para o aluno (MARTINS, 2010; TESTONI, 2004; KAMEL, 2006).

\section{Considerações finais}

Mesmo a Astronomia possuindo um caráter interdisciplinar e tendo seus conteúdos indicados pelos documentos oficiais, as pesquisas mostram que a mesma não está sendo trabalhada de maneira adequada no ensino médio e nem nos cursos de formação de professores. Visando contribuir neste sentido, o presente trabalho, embasado na aprendizagem significativa, propõe a construção de uma atividade didática experimental que simula as fases da Lua e verifica o processo de construção dos conceitos envolvidos neste tema por meio da elaboração de Histórias em Quadrinhos pelos próprios alunos. Além de sua aplicação direta em sala de aula, o uso desta proposta está sendo estudada (atualmente em fase de coleta de dados) em uma amostra de professores em processos de formação continuada em um ambiente não-formal de ensino de Astronomia. Portanto, acreditamos que, além de proporcionar uma aula diferenciada em relação às chamadas "aulas tradicionais", tais ações poderão potencializar o ensino da Astronomia apoiado nos princípios psicolinguísticos, cognitivos e da ludicidade, segundo apresentado na fundamentação.

\section{Referências}

ALBRECHT, E.; VOELZKE, M.R. Construção de Historias em Quadrinho nas aulas de física: Uma prática didática. In: VII Encontro nacional de pesquisa em educação em ciências, 2009, Florianópolis. Anais. Florianópolis: UFSC, 2009.

ANDRADE, M. J. P. et al. Investigando conhecimento básico em astronomia de professores em formação. In: VII Encontro nacional de pesquisa em educação em ciências, 2009, Florianópolis. Anais. Florianópolis: UFSC, 2009.

BAILEY, J. M.; SLATER, T. F. A Review of Astronomy Education Research. Astronomy Education Review, v.2, p.20-45, 2004.

BRASIL. Parâmetros Curriculares Nacionais para o Ensino Médio. Brasília: Secretaria de Educação Média e Tecnologia, 1999.

BRASIL. Parâmetros Curriculares Nacionais para o Ensino Médio- Ciências da Natureza, Matemática e suas Tecnologias. Brasília: Secretaria de Educação Média e Tecnologia, 2002.

CAMINO, N. Aprender a imaginar para comenzar a comprender. Revista Alambique. [versión electrónica], 42, 2004. 
DARROZ, L. M.; HEINECK, R.; PÉREZ, C. A. S. Conceitos básicos de Astronomia: uma proposta metodológica. Revista Latino-Americana de Educação em Astronomia, n. 12, p. 57-69, 2011.

DARROZ, L. M.; PÉREZ, C. A. S.; ROSA, C. W.; HEINECK, R. Propiciando aprendizagem significativa para alunos do sexto anos do ensino fundamental: um estudo sobre as fases da Lua. Revista Latino-Americana de Educação em Astronomia, n. 13, p. 31-40, 2012.

GONÇALVES, R.; MACHADO, D. M. Comics: investigación de conceptos y de términos paleontológicos, y uso como recurso didáctico en la educación primaria. Enseñanza de las Ciências, Barcelona, v. 23, n. 2, p. 263-274, 2005.

IACHEL, G.; LANGHI, R.; SCALVI, R. M. F. As fases da lua e as concepções alternativas de alunos do ensino médio. In: VI Encontro nacional de pesquisa em educação em ciências, 2007, Florianópolis. Anais. Florianópolis: UFSC, 2007.

JACKSON, E. Practical astronomical activities during daytime. Revista LatinoAmericana de Educação em Astronomia, n.8, p. 71-88, 2009.

KAMEL, C. R. L. Ciências e quadrinhos: explorando as potencialidades das histórias como materiais instrucionais. 113f. Dissertação (Mestrado em Ensino em Biociências e Saúde), Instituto Oswaldo Cruz - Fiocruz, Rio de Janeiro: 2006.

KANTOR, C. A. A ciência do céu: uma proposta para o ensino médio. São Paulo: USP/IF/SBI-037/2001. Dissertação (Mestrado) - Universidade de São Paulo. Instituto de Física. Departamento de Física Experimental. São Paulo, 2001.

LANGHI, R.; NARDI, R. Formação de professores e seus saberes disciplinares em astronomia essencial nos anos iniciais do ensino fundamental. Revista Ensaio, v.12, n.2, p.205-224, 2010.

LANGHI, R. Astronomia nos anos iniciais do ensino fundamental: repensando a formação de professores. 2009. 370 f. Tese (Doutorado em Educação para a Ciência). Faculdade de Ciências, UNESP, Bauru.

LANGHI, R. Aprendendo a ler o céu: pequeno guia prático para a astronomia observacional. Campo Grande, MS: Ed. UFMS, 2011.

LEÃO, D. S. Mini-planetário: um projetor portátil de baixo custo. Física na Escola, v. 12, n. 2, 2011.

LONGHINI, M. D.; SILVESTRE, R. F.; VIEIRA, F. C. F. Uma estratégia para construção de rosa dos ventos envolvendo geometria, arte, astronomia e tecnologia. Física na Escola, v. 11, n. 1, 2010.

LONGHINI, M. D. (org.). Educação em Astronomia: experiências e contribuições para a prática pedagógica. Campinas, SP: Átomo, 2010.

MARRANGHELLO, G. F.; PAVANI, D. B. Utilizando a câmera fotográfica digital como ferramenta para distinguir as cores das estrelas. Física na Escola, v. 12, n. 1, 2011.

MARTINS, B. A.; LANGHI, R. Aprendizagem significativa na elaboração de histórias em quadrinhos sobre astronomia por alunos do ensino médio. In: I Simpósio Nacional de Educação em Astronomia, 2011, Rio de Janeiro. Anais. Rio de Janeiro: UERJ,2011. 
MARTINS, B. A. Um estudo sobre a construção de histórias em quadrinhos por alunos do ensino médio como uma estratégia para o ensino das leis de Newton. 2010. Trabalho de Conclusão de Curso (Licenciatura em Física). Centro de Ciências Exatas e Tecnologia, UFMS, Campo Grande. Disponível em: https:<//sites.google.com/site/proflanghi/tcc201004>. Acesso em: 28 Out. 2011.

MOREIRA, M. A.; MASINI, E.S. Aprendizagem significativa: A teoria de David Ausubel. São Paulo: Ed. Moraes, 1982, 7-52 p.

PELIZZARI, A; et al. Teoria de aprendizagem significativa segundo Ausubel. Revista PEC, Curitiba, 2001- 2002. V. 2, n.1, p.37-42.

PRICE, A. The Astronomy Education Research Charter. Astronomy Education Review, 8 (1), 010601, 2009.

QUEIROZ, G. P.; SOUZA, C. J. B.; MACHADO, M. A. D. A prática de pesquisa de um professor do ensino fundamental envolvendo modelos mentais de fases da Lua e eclipses. Revista Latino-Americana de Educação em Astronomia, n.8, p. 19-36, 2009.

ROS, R. M. Estudio del horizonte local. Revista Latino-Americana de Educação em Astronomia, n.8, p. 51-70, 2009.

ROSA, P. R. S. Instrumentação para o ensino de ciências. Campo Grande: Ed. UFMS, 2008.

SARAIVA, M. F.; AMADOR, C. B.; KEMPER, E.; GOULART, P.; MULLER, A.As fases da Lua numa caixa de papelão. Revista Latino-Americana de Educação em Astronomia, n.4, p.9-26, 2007.

SILVA, G. S. F.; VILLANI, A. O processo grupal nas aulas de física: A análise do grupo da resistência. XI Encontro de Pesquisa em Ensino de Física. Curitiba, 2008.

SILVEIRA, F. L. As variações dos intervalos de tempo entre as fases principais da Lua. Revista Brasileira de Ensino de Física, 23(3), p.300-307, set. 2001.

SILVEIRA, F. L. Marés, fases principais da Lua e bebês. Caderno Brasileiro de Ensino de Física, v.20, n.1, p.10-29, abr. 2003.

TESTONI, L. A. Um corpo que cai: as Histórias em Quadrinhos no Ensino de Física. Dissertação de mestrado. FEUSP, 2004.

TESTONI, L. A.; ABIB, M. L. V. S. A utilização de histórias em quadrinhos no ensino de física: uma proposta para o ensino de inércia. Enseñanza de las Ciencias, Granada, v. extra, p. 1-5, 2005.

VÍLCHEZ-GONZÁLEZ, J. M.; PERALES-PALACIOS, F. J. Image of science in cartoons and its relationship with the image in comics. Physics Education, v. 41, p. 240-249, 2006.

WERLANG, R. B.; SCHNEIDER, R. S.; SILVEIRA, F. L. Uma experiência de ensino de física de fluidos com o uso de novas tecnologias no contexto de uma escola técnica. Revista Brasileira de Ensino de Física, v.30, nº1, 2008, São Paulo.

WORNER, C. H.; ROMERO, A. Una manera diferente de enseñar física: Física y humor. Enseñanza de las Ciências, Barcelona, v. 16, n. 1, p. 187-192. 1998. 